\title{
Monte Carlo Simulation of Smectic Liquid Crystals and the Electroclinic Effect: the Role of the Molecular Shape
}

\author{
Jianling Xu and Robin L. B. Selinger* \\ Physics Department, Catholic University of America, Washington, DC 20064 \\ Jonathan V. Selinger, B. R. Ratna, and R. Shashidhar \\ Center for Bio/Molecular Science and Engineering, Naval Research Laboratory, Code 6900, \\ 4555 Overlook Avenue, SW, Washington, DC 20375
}

(July 27, 1999)

\begin{abstract}
Using Monte Carlo simulation methods, we explore the role of molecular shape in the phase behavior of liquid crystals and the electroclinic effect. We study a "bent-rod" mesogen shaped like the letter Z, composed of seven soft spheres bonded rigidly together with no intra-molecular degrees of freedom. For strongly angled molecules, we find that steric repulsion alone provides the driving force for a smectic-C phase, even without intermolecular dipole-dipole interactions. For weakly angled (nearly rod-like) molecules, we find a stable smectic-A (SmA) phase and a strong electroclinic effect with a saturation tilt angle of about $19^{\circ}$. In the SmA phase we find evidence of vortex-like point defects. We also observe a field-induced nematic-smectic phase transition.

PACS numbers: 61.30.Cz, 64.70.Md, 61.30.Gd, 61.30.Jf
\end{abstract}

\section{INTRODUCTION}

The response of smectic liquid crystals to applied electric fields has been extensively studied for both basic research and applications. One subject of particular interest is the electroclinic effect, which occurs in the smecticA $(\mathrm{SmA})$ phase of chiral molecules. In the electroclinic effect, an applied electric field in the smectic layer plane induces a tilt of the molecules relative to the layer normal, in a direction orthogonal to the field. The magnitude of the induced tilt scales linearly with the applied electric field for low fields, and then saturates at higher fields. This effect was predicted by Meyer on the basis of symmetry [1], and it was subsequently observed experimentally by Garoff and Meyer [2]. It is now being exploited for electro-optic devices that display a continuous gray scale as a function of applied electric field, such as spatial light modulators [3, 3 .

To optimize electroclinic liquid crystals for device development, one needs a theoretical understanding of how the electroclinic tilt depends on electric field, temperature, and molecular structure. So far, most theoretical work on the electroclinic effect has been through Landau theory, i.e. a minimization of the free energy expanded in powers of the molecular tilt and the electrostatic polarization [1, [5],6]. This work explains certain aspects of the electroclinic effect-in particular, it shows how the tilt and polarization depend on field for low fields, and it shows how the susceptibility to a field increases as the system approaches the second-order phase transition from the SmA to the smectic-C (SmC) phase. However, some important questions about the electroclinic effect are not addressed by Landau theory. The first and most general question is: How sensitive is the electroclinic ef- fect to molecular shape? In other words, how much does the electroclinic susceptibility change with slight details of molecular structure? A second and more specific question is: How does the applied electric field change the distribution of molecular orientations? Does it make the molecules tilt as rigid rods from an initially untilted state to a tilted state? Or does it change a state of disordered tilt in random directions into a state of ordered tilt in one direction? The latter alternative is suggested by the de Vries description of the SmA phase [7].

To address these questions, in this paper we present a series of Monte Carlo simulations of smectic liquid crystals. Simulation is an appropriate tool to address these questions for two reasons. First, in simulations we can begin with a microscopic model for the molecular structure and determine the large-scale order of the liquidcrystal system as a function of thermodynamic variables such as temperature, density, and applied field. We can then make small changes in the molecular shape and see how these changes affect the large-scale order of the system. Thus, we can determine how macroscopic properties such as the electroclinic susceptibility depend on details of the molecular shape. Second, in simulations we can take snapshots of the positions and orientations of all the molecules in the system, and hence can extract any correlation function to characterize the system. This information is not available in Landau theory, and is generally difficult to extract from experiments. Hence, simulations give us new information about the distribution of molecular orientations as a function of electric field, and about topological defects in the molecular orientations.

In these simulations, we use a "bent-rod" rigid molecule with the oblique shape shown in Fig. 1. This shape is motivated by three considerations. First, the three-dimensional structure of many liquid-crystal 
molecules, such as the homologous series KNnm, has this general shape [8]. In the center is a rigid molecular core, which defines the optical axis of the molecule, and on both ends are hydrocarbon chains, which extend out at an angle from the core. In the homologous series $\mathrm{KNnm}$, the electroclinic tilt angle of the SmA phase can be increased by making the hydrocarbon chains longer, thus making the molecules more oblique. Second, the Boulder model for ferroelectric liquid crystals shows that molecules in the SmC phase typically take the shape of bent cylinders [9]. For that reason, we can regard this shape as a generic feature of smectic liquid crystals. Third, density functional theory has been used to predict the phase diagram of parallel offset hard cylinders, a shape similar to bent rods [10]. That work showed a high-density SmC phase for molecules with a higher offset ratio, i.e. the more oblique. These results confirm that the obliqueness of molecular shape is an important parameter to determine the phase behavior of smectic liquid crystals.

To simulate a simple molecular structure with a bentrod shape of variable obliqueness, we use a molecule composed of seven spheres arranged in the shape of the letter $\mathrm{Z}$, as illustrated in Fig. 1. The spheres are "glued" rigidly together with no intra-molecular degrees of freedom, with a bend angle $\theta$ between the core and tail portions of the molecule. We consider the cases $\theta=45^{\circ}$, which is quite oblique, and $\theta=5^{\circ}$, which approaches the rod-shaped limit of $\theta=0^{\circ}$. Each molecule also has a dipole moment that lies perpendicular to the molecular backbone, as shown, giving the molecule a chiral structure. The molecules interact through a soft repulsive sphere-sphere pair potential, and each molecular dipole interacts with the applied electric field. We neglect dipole-dipole interactions as an approximation to simplify the computations.

These simulations provide clear evidence that steric repulsion alone can give rise to order in the molecular tilt, even without including intermolecular dipole-dipole interactions. Furthermore, they show that the bend angle $\theta$ plays a major role in determining phase behavior. For $\theta=45^{\circ}$ the system has a phase transition directly from the isotropic phase to the SmC phase. By contrast, for $\theta=5^{\circ}$, the system has nematic and SmA phases, each stable over a wide range of temperature. In the absence of an applied electric field, the molecules of the SmA phase are not aligned with the layer normal but rather are tilted in random directions, and the orientation of the tilt exhibits vortex-like point defects. When an electric field is applied, the magnitude of the molecular tilt increases and the direction of the tilt becomes more ordered, giving a strong electroclinic effect. At high fields, the electroclinic tilt angle saturates at approximately $19^{\circ}$. The simulations also show that a high electric field applied to the nematic phase induces a transition into the SmA phase, showing another ordering effect of the field.

The plan of this paper is as follows. In Sec. II we describe the details of the model and the computational method that we used. In Sec. III we present the results of the simulations for bend angle $\theta=45^{\circ}$ and $\theta=5^{\circ}$. In particular, we show the electroclinic effect in the $\mathrm{SmA}$ phase for $\theta=5^{\circ}$. Finally, in Sec. IV, we discuss the significance of these results for experiments on smectic liquid crystals.

\section{SIMULATION METHOD}

In our simulations, we consider molecules composed of seven soft spheres arranged in the rigid bent structure shown in Fig. 1. The molecular director is defined as the unit vector along the five-sphere core of the molecule. The interaction between molecules is reduced to an interaction between different spheres in different molecules. Intramolecular interactions and degrees of freedom are suppressed. The sphere-sphere interaction potential is the truncated Lennard-Jones potential, also known as the Weeks-Chandler-Anderson potential [1], cut off at its minimum so there is no attractive tail:

$U_{m n}^{\mathrm{int}}= \begin{cases}4 \epsilon\left[\left(\frac{\sigma}{r_{m n}}\right)^{12}-\left(\frac{\sigma}{r_{m n}}\right)^{6}\right]+\epsilon, & \text { if } r_{m n} \leq r_{c}=2^{1 / 6} \sigma \\ 0, & \text { otherwise. }\end{cases}$

where $r_{m n}=\left|\vec{r}_{m}-\vec{r}_{n}\right|$ and $m$ and $n$ are the sphere indices in different molecules. We choose this short-range repulsive interaction to reduce required computation time and to focus on the role of steric effects without any contribution from attractive interactions. For the rest of this paper, we measure lengths in units of $\sigma$ and energies in units of $\epsilon$. In addition, each molecule interacts with the applied electric field $\vec{E}$ through the coupling

$$
U_{j}^{\text {dipole }}=-\vec{E} \cdot \vec{p}_{j},
$$

where $\vec{p}_{j}$ is the dipole moment of molecule $j$. The molecular dipole moment is defined to have unit magnitude, which gives a scale for the electric field. We simulate 500 molecules in a flexible three-dimensional box with periodic boundary conditions. We keep the system with constant volume density 0.75 Lennard-Jones particles per unit volume, and allow the aspect ratio of the simulation cell to adjust according to the Metropolis algorithm. We do not allow the cell to shear.

The system is prepared by a procedure analogous to the experimental technique of cooling in a strong aligning field to avoid the formation of smectic domains. We begin the simulations at the high temperature $k_{B} T=20.0$, with the box size of $11.5 \times 11.5 \times 35.0$. This aspect ratio favors the formation of a five-layered smectic phase. In the initial state, the molecules have random positions but all the molecules are "double-aligned," that is, both the directors and the dipole moments are aligned. During the preliminary cooling procedure, we suppress all orientational degrees of freedom and allow the molecules to 
diffuse while remaining double-aligned. The temperature of the system is reduced slowly at a rate $10^{-4}$ per Monte Carlo step. The system comes to equilibrium quickly. In about 10,000 Monte Carlo steps, the molecules form five distinct layers. If the layer normal is not parallel to the $z$ axis, we measure the angle away from the $z$ axis, adjust the director of the molecules, choose a new random initial configuration, and repeat the simulation to get a layered system with the layer normal along the $z$ axis, with no defects in the layer structure.

Once we reach this double-aligned smectic state, we reduce the temperature to about 1.5, still in the doublealigned state. Then, after the system is in equilibrium, we switch on the three rotational degrees of freedom for each molecule and equilibrate for an additional 100,000 Monte Carlo steps per particle. In one Monte Carlo step, each randomly selected molecule attempts three translations and three rotations.

To characterize the phase behavior of the system, we particularly use three order parameters. First, the nematic order tensor $Q$ represents the strength and direction of orientational order of the molecules. It is defined as

$$
Q_{\alpha \beta}=\left\langle\frac{1}{N} \sum_{j=1}^{N}\left(\frac{3}{2} n_{j \alpha} n_{j \beta}-\frac{1}{2} \delta_{\alpha \beta}\right)\right\rangle,
$$

where $\vec{n}_{j}$ is the director along the core of molecule $j$ and $N=500$ is the number of molecules. The eigenvector corresponding to the maximum eigenvalue of $Q$ is the average director of the system. If the eigenvalue is 1 , the molecular directors are completely aligned; if the value is lower it reflects less perfect alignment. Second, the polarization $\vec{P}$ represents the degree of orientation of the molecular dipole moments. It is defined as the vector average

$$
\vec{P}=\left\langle\frac{1}{N} \sum_{j=1}^{N} \vec{p}_{j}\right\rangle .
$$

Third, the smectic order parameter $\sigma$ represents the strength of the density modulation along the $z$ direction. It is defined as

$$
\sigma=\left\langle\frac{1}{N} \sum_{j=1}^{N} e^{2 \pi i z_{j} / d}\right\rangle,
$$

where $z_{j}$ is the $z$-coordinate of the center of mass of molecule $j$ and $d$ is the smectic layer wavelength, which is one-fifth of the $z$-dimension of the simulation cell.

\section{RESULTS}

\section{A. Forty-Five Degree Bent-Rod Molecules}

For the molecules with the large bend angle $\theta=45^{\circ}$, the phase sequence is crystal-SmC-isotropic. The $\mathrm{SmC}$ phase is stable over a wide range of temperature, from approximately $k_{B} T=0.5$ to 1.5 (in Lennard-Jones units). A sample configuration is shown in Fig. 2. The polarization of any single layer, defined by Eq. (4), is $P=0.85$, indicating nearly perfect orientational order.

In spite of the high orientational order within each layer, the local tilt direction of each layer is only loosely coupled to that of adjacent layers, and it tends to wander. This type of behavior was evident also in the simulation study of Affouard et al. 12 on a related system. It is similar to the proposed random smectic- $\mathrm{C}_{\mathrm{R}}$ phase, which has been suggested as a model for the thresholdless switching observed experimentally in certain smectic liquid crystals [13. An alternative model has recently been proposed for these experiments 14, but the smectic- $C_{R}$ phase remains a theoretical possibility for future materials. Indeed, this proposed phase with random orientations of adjacent layers can be viewed as one version of the sliding phase that has been investigated in recent theoretical work 15.

One possible explanation for the low interlayer correlations in our simulations is that there is very little interaction between the tilt directions in adjacent layers, because the intermolecular potential is purely repulsive and because there is hardly any interdigitation between the layers. As a result, the adjacent layers should have very little preference for synclinic (ferroelectric) or anticlinic (antiferroelectric) order, and they should be fairly free to wander between these extremes. An alternative explanation is that the layers might prefer anticlinic order, but they are frustrated because the system has an odd number of layers (five). It is interesting to note that Affouard's simulation also included an odd number of layers (three). This latter explanation seems less likely, however, because the interaction between layers does not seem to favor anticlinic order.

Note that we observe the SmC phase even though we have not included dipole-dipole interactions in our intermolecular potential, indicating that steric repulsion defined by molecular shape is sufficient to produce order in the molecular tilt direction. Electrostatic interactions are not required to produce a tilted smectic [16]. Presumably the inclusion of dipole-dipole interactions in our simulation would increase the temperature range over which the $\mathrm{SmC}$ is stable, and it would likely increase the coupling between the tilt directions in adjacent layers.

\section{B. Five Degree Bent-Rod Molecules}

The molecule with bend angle $\theta=5^{\circ}$ looks very nearly like a rod, but it has properties quite different from a purely rod-shaped molecule. The $\theta=5^{\circ}$ system has a stable SmA phase over a temperature range of $k_{B} T=$ 0.7 to 3.0. A sample configuration of the $\mathrm{SmA}$ phase is shown in Fig. 3a. The smectic order parameter defined by Eq. (5) is very high, about 0.9 . 
The molecules in each layer of the SmA phase have approximately zero average tilt and no net polarization. However, a close look at the structure of an individual layer shows that the local molecular tilt is nonzero but that defects cause the net tilt to vanish, as shown in Fig 3b. In some configurations, these point defects in the local tilt appear to be vortices analogous to those seen in, for example, an $x y$ model 117. Comparison of defect structures in adjacent layers shows that there is no strong correlation in defect location between layers, indicating that these defects are truly point vortices and do not thread through all five layers of the system. In this respect, they are analogous to the "pancake" vortices seen in layered superconductors with weak interlayer coupling [18].

When we apply an electric field in the SmA layer plane, the molecules tilt showing a clear electroclinic effect. The observed polarization responds rapidly to the applied field, coming close to its equilibrium value in only several thousand Monte Carlo steps, while the tilt angle takes up to 500,000 Monte Carlo steps to equilibrate. This equilibration would likely have been faster if we had implemented degrees of freedom that allowed shear deformation of the simulation cell, but clearly it is much longer than the equilibration time for the polarization. Figure 4a shows the SmA phase under a strong applied field $E=10$. When we examine a layer from this system, we observe that the vortex-like defects have vanished, and the molecules in the same layer are all closely aligned, as shown in Fig. 4b.

We can compare the measured polarization response to the applied field with the prediction of a simple spin model. The molecules in a smectic layer are localized with directors pointing in almost the same direction. The most active movement is the rotation of the molecular dipole moment around the director. In view of this property, we consider the molecules as two-dimensional independent dipoles with only one effective rotational degree of freedom. The net polarization can then be written as

$$
P=\frac{\int_{0}^{2 \pi} p \cos \theta e^{E p \cos \theta / k_{B} T} d \theta}{\int_{0}^{2 \pi} e^{E p \cos \theta / k_{B} T} d \theta}=\frac{I_{1}\left(E p / k_{B} T\right)}{I_{0}\left(E p / k_{B} T\right)}
$$

where $I_{0}$ and $I_{1}$ are modified Bessel functions and $p=1$ is the magnitude of the dipole moment of a single molecule. The simulation results for polarization vs. field are plotted together with the analytic prediction in Fig. 5a, and are in close agreement. Indeed, the agreement is much closer than one would expect from such a simple modelone would expect the interactions among the molecules to give collective order that would give a higher initial slope to the the polarization vs. field curve. This agreement shows that the alignment of molecular dipole moments with the electric field is a single-molecule effect rather than a collective effect for these nearly rod-like molecules. Collective effects should become more important if we increase the interaction between the directions of the molecular dipoles - either by including dipole-dipole in- teractions in our simulation model or by increasing the bend angle $\theta$ to make the molecules more oblique.

In addition to these results for the polarization, we also measure the molecular tilt angle in the simulations. The average tilt angle for the system is extracted from the nematic order tensor $Q$ defined by Eq. (3). From the eigenvector corresponding to the maximum eigenvalue of $Q$, we can calculate the tilt angle away from the layer normal. Using this technique, we measure the tilt angle as a function of applied electric field in the simulation for two temperatures, $k_{B} T=0.7$ and $k_{B} T=1.3$. The results are shown in Fig. 5b. We observe that the tilt angle responds more sharply to the applied field at lower temperature than at higher temperature; that is, the electroclinic coefficient drops with increasing temperature. This temperature dependence is similar to the temperature dependence of the electric susceptibility shown in Fig. 5a. At high applied field, the tilt angle saturates at about $19^{\circ}$ for both temperatures. We note that the tilt angle, in contrast with the polarization, is a collective effect rather than a single-molecule effect in this simulation, as shown by the much longer equilibration time for the tilt angle. Thus, the saturated tilt angle of $19^{\circ}$ is not simply related to the molecular geometry, but depends on the collective order of many molecules whose transverse dipoles have been aligned by the applied electric field.

When the molecules tilt under an electric field, the thickness of the smectic layers shrinks. Because the simulation cell is flexible, the $z$-dimension of the cell also shrinks. At the temperature $k_{B} T=0.7$, the $z$-dimension of the cell changes from 36.7 at $E=0$ to 35.0 at $E=10$, which is a contraction by a factor of 0.954 . This contraction is analogous to the change in the smectic layer spacing under an electric field observed in x-ray diffraction experiments [19], and the contraction factor can be interpreted as the cosine of an x-ray tilt angle of $17.5^{\circ}$. This x-ray tilt angle is somewhat smaller than the tilt angle of $19^{\circ}$ associated with the eigenvectors of $Q$, which corresponds to the orientational ordering of the molecular cores observed in optical experiments.

\section{Field-Induced Phase Transition}

We carried out further studies of the five-degree molecular system in a larger temperature range, and located the SmA-nematic transition at approximately $k_{B} T=$ 3.0. Above that temperature, the SmA phase melts and the system is stable as a nematic state, with low positional correlations (smectic order parameter below 0.3) but with very high orientational order. Figure 6 a shows a slice of the nematic system at $k_{B} T=3.3$, viewed from the $x$ direction. When the temperature is lowered from $k_{B} T=3.3$ to $k_{B} T=2.1$, the system returns to the $\mathrm{SmA}$ phase with clearly defined layers (smectic order parameter of about 0.8 ). This is evidence that the system has a 
stable and reversible SmA-nematic phase transition.

Under a strong electric field, the nematic phase has a surprising behavior. We apply $E=10$ to the nematic system at $k_{B} T=3.3$, not far above the nematic-SmA transition temperature. The system regains a large smectic order parameter and again forms clearly defined layers, as shown in Fig. 6b. This figure shows a slice of the system, with five layers in cross section. Thus we observe in this simulation a field-induced nematic-SmA phase transition. In experiments, electric-field-induced isotropicnematic-smectic phase transitions have been observed in thermotropic liquid crystals [20], and the critical behavior of the field-induced molecular tilt near the nematicSmA transition has been investigated 21. A good understanding of these effects in simulation will contribute to a better understanding of field-induced phase transitions in experiment.

\section{DISCUSSION}

This simulation study shows that the molecular shape is very important for the phase behavior of liquid crystals. In the system with the $45^{\circ}$ molecular bend angle, the steric repulsion based on molecular shape provides the driving force for molecular tilt order in a SmC phase, even without intermolecular dipole-dipole interactions. In the system with the $5^{\circ}$ bend angle, the molecules are closer to rigid rods, so they do not exhibit a $\mathrm{SmC}$ phase with spontaneous tilt order. Still, even a $5^{\circ}$ molecular bend leads to a substantial electroclinic effect, which would be totally absent for rigid rods. (Rigid rods with transverse electric dipoles would align their dipoles with an applied electric field, but this alignment would not lead to any molecular tilt.) Preliminary simulation results for molecules with a $9^{\circ}$ bend angle (not presented here) suggest that the phase transitions shift dramatically from the $5^{\circ}$ molecules, confirming the influence of small changes in molecular shape. Hence, one conclusion of this study is that collective intermolecular properties like molecular tilt and transition temperatures are quite sensitive to slight details of molecular shape. This conclusion is somewhat disappointing from the perspective of modeling unique properties of particular liquid-crystal compounds, as opposed to generic properties based on molecular symmetry, because it implies that one must describe the molecular structure very precisely in order to predict properties like tilt and transition temperatures.

Another conclusion of this study is that the distribution of molecular tilts in the SmA phase is more complex than is often supposed. In the absence of an applied electric field, the molecules do not stand up as rigid rods along the layer normal. Rather, there is disorder in the molecular tilt, with all of the molecules tilting away from the layer normal in random azimuthal directions. Some of this disorder takes the form of vortices in the tilt projected into the smectic layer plane. When an electric field is applied, it has two effects: it increases the magnitude of the tilt angles and it increases the order in the azimuthal direction of the tilt. These two effects combine to give the electroclinic tilt angle associated with the eigenvectors of the nematic order tensor $Q$. For that reason, this tilt angle is somewhat greater than the x-ray tilt angle associated with the contraction of the smectic layers, which arises only from the increase in the magnitude of the molecular tilt angles. This result suggests that experimental measurements of the electroclinic effect cannot be interpreted purely as tilting of rigid rods or as ordering of $x y$ spins, but rather as a combination of both.

The vortices observed in the SmA phase of the simulation are particularly intriguing defects. These vortices appear to be equivalent to the topological defects that mediate the Kosterlitz-Thouless ordering transition in the two-dimensional $x y$ model [17]. Thus, they suggest that the SmA phase is analogous to the disordered phase of the $x y$ model and the SmC phase to the ordered phase. It is surprising that our three-dimensional simulation shows point vortices that are uncoupled from one smectic layer to the next, and do not thread through all five layers of the system. This uncoupling presumably occurs because, as noted earlier, there is very little interaction between the tilt directions in adjacent layers due to the short-range repulsive potential and the lack of interdigitation between layers. The observation of these defects leads to several questions for future research. For example, how do the defects evolve as a small electric field is applied? In a system with a SmA-SmC transition, what happens to the defects when the temperature drops toward the transition? Furthermore, if the interaction between molecules had a longer range, would the point-like "pancake" vortices turn into vortex lines as in conventional type II superconductors [18], or would they be driven out of the system completely? This final question is a key issue for experimental systems in which the tilt directions of adjacent layers are strongly coupled.

In summary, we have simulated smectic ordering in liquid crystals composed of bent-rod molecules interacting through a soft repulsive potential. The system of highly bent molecules shows a SmC phase with spontaneous tilt ordering, while the system of only slightly bent molecules shows a SmA phase with a substantial induced tilt under an applied electric field. These results show the high sensitivity of molecular tilt ordering to the molecular shape, and show the distribution of molecular tilt that controls the electroclinic effect.

\section{ACKNOWLEDGMENTS}

This work was supported by the U. S. Navy Grant No. N00014-97-1-G003, the National Science Foundation Grant No. DMR-9702234-1, and the Donors of the Petroleum Research Fund, administered by the American 
Chemical Society.

[1] R. B. Meyer, Mol. Cryst. Liq. Cryst. 40, 33 (1977).

[2] S. Garoff and R. B. Meyer, Phys. Rev. Lett. 38, 848 (1977).

[3] N. Collings, W. A. Crossland, R. C. Chittick, and M. F. Bone, Proc. SPIE Int. Soc. Opt. Eng. 963, 46 (1989).

[4] G. Andersson, I. Dahl, L. Komitov, S. T. Lagerwall, K. Skarp, and B. Stebler, J. Appl. Phys. 66, 4983 (1989).

[5] T. Carlsson, B. Žekš, C. Filipič, A. Levstik, and R. Blinc, Mol. Cryst. Liq. Cryst. 163, 11 (1988); B. Žekš and R. Blinc, in Ferroelectric Liquid Crystals (Gordon and Breach, Philadelphia, 1991), p. 365.

[6] I. Abdulhalim and G. Moddel, Liquid Crystals 9, 493 (1991).

[7] A. de Vries, A. Ekachai, and N. Spielberg, Mol. Cryst. Liq. Cryst. Lett. 49, 143 (1979); A. de Vries, Mol. Cryst. Liq. Cryst. Lett. 49, 179 (1979).

[8] For reviews, see B. R. Ratna, G. P. Crawford, S. K. Prasad, J. Naciri, P. Keller, and R. Shashidhar, Ferroelectrics 148, 425 (1993); G. P. Crawford, J. Naciri, R. Shashidhar, P. Keller, and B. R. Ratna, Mol. Cryst. Liq. Cryst. A 263, 223 (1995). For the three-dimensional structure of KN125, see A. Hermanns, C. Wilson, J. Patel, K. Grüneberg, K. Nelson, A. Townsend-Booth, and B. Ratna, Proc. SPIE Int. Soc. Opt. Eng. 3297, 73 (1998).

[9] D. M. Walba, H. A. Razavi, A. Horiuchi, K. F. Eidman, B. Otterholm, R. C. Haltiwanger, N. A. Clark, R. Shao, D. S. Parmar, M. D. Wand, and R. T. Vohra, Ferroelectrics 113, 21 (1991).

[10] A. M. Somoza and P. Tarazona, J. Chem. Phys. 91, 517 (1989).

[11] J. D. Weeks, D. Chandler, and H. C. Andersen, J. Chem. Phys. 54, 5237 (1971); D. Chandler, J. D. Weeks, and H. C. Andersen, Science 220, 787 (1983).

[12] F. Affouard, M. Kroger, and S. Hess, Phys. Rev. E 54, 5178 (1996).

[13] S. Inui, N. Iimura, T. Suzuki, H. Iwane, K. Miyachi, Y. Takanishi, and A. Fukuda, J. Mater. Chem. 6, 671 (1996).

[14] P. Rudquist, J. P. F. Lagerwall, M. Buivydas, F. Gouda, S. T. Lagerwall, N. A. Clark, J. E. MacLennan, R. Shao, D. A. Coleman, S. Bardon, T. Bellini, D. R. Link, G. Natale, M. A. Glaser, D. M. Walba, M. D. Wand, and X.-H. Chen, J. Mater. Chem. 9, 1257 (1999).

[15] C. S. O'Hern, T. C. Lubensky, and J. Toner, preprint cond-mat/9904415.

[16] M. A. Glaser, R. Malzbender, N. A. Clark, and D. M. Walba, J. Phys. Condens. Mat. 6, A261 (1994).

[17] D. R. Nelson, in Phase Transitions and Critical Phenomena, Vol. 7, edited by C. Domb and J. L. Lebowitz (Academic Press, New York, 1983).

[18] G. W. Crabtree and D. R. Nelson, Phys. Today 50 (4), 38 (1997).
[19] G. P. Crawford, R. E. Geer, J. Naciri, R. Shashidhar, and B. R. Ratna, Appl. Phys. Lett. 65, 2937 (1994).

[20] I. Lelidis and G. Durand, Phys. Rev. Lett. 73, 672 (1994).

[21] S.-D. Lee and J. S. Patel, Phys. Lett. A 155, 435 (1991). 


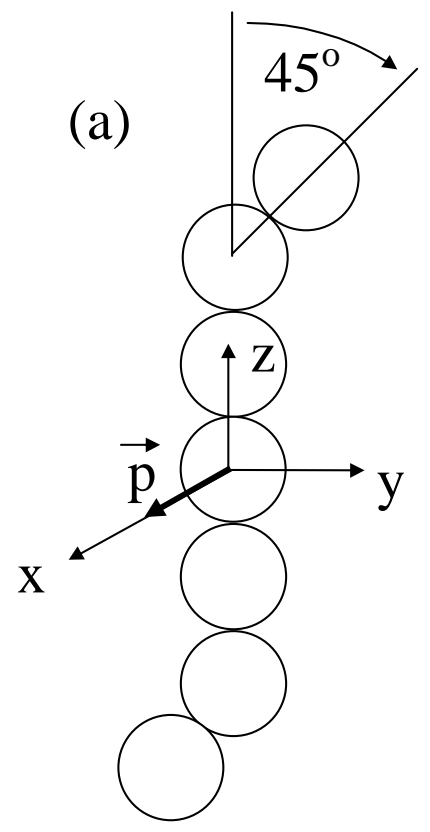

(b)

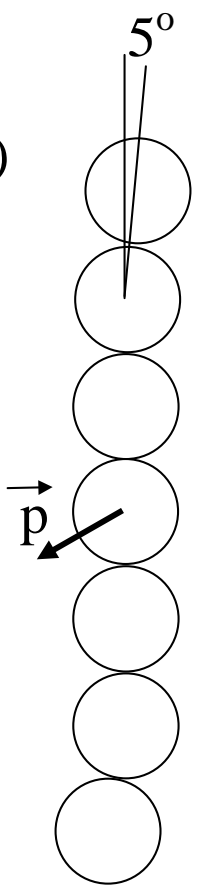

FIG. 1. Basic molecular shape, with the bend angle $\theta$ between the core and tail portions of the molecule.

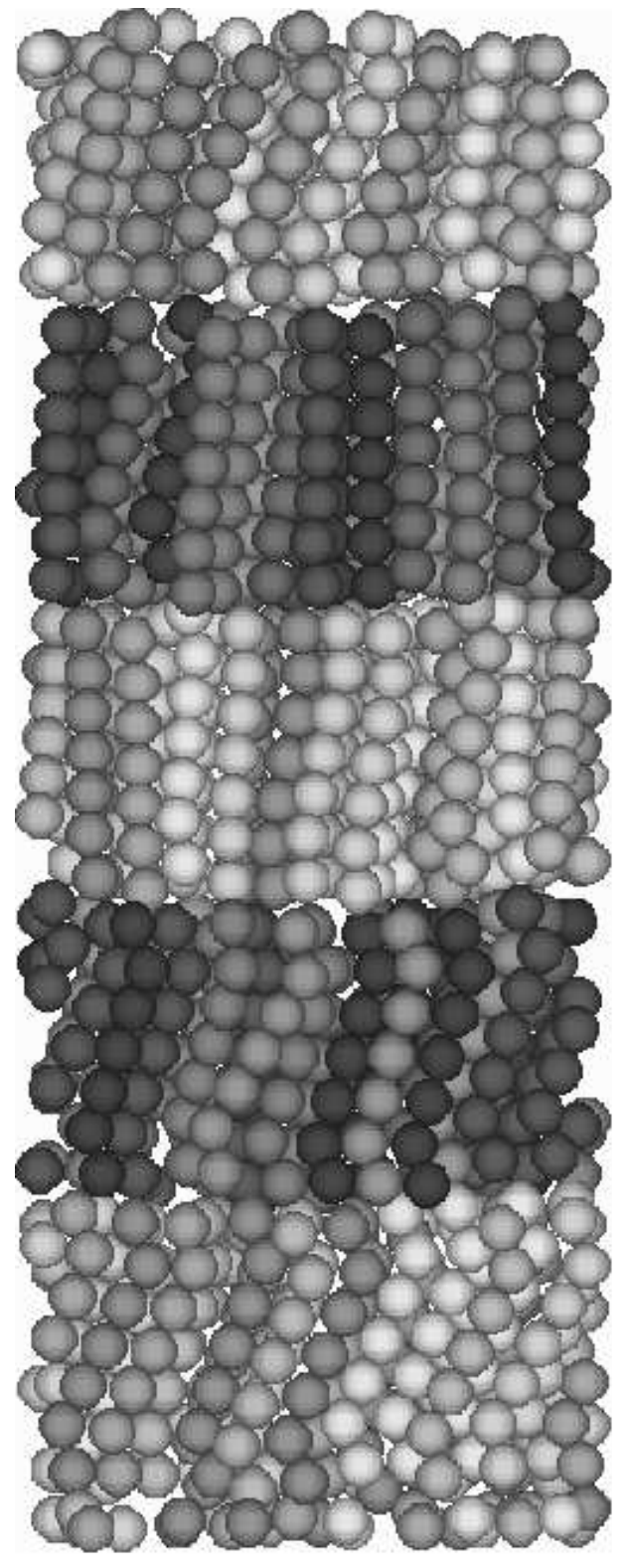

FIG. 2. For the molecules with bend angle $\theta=45^{\circ}$, the simulations show a SmC phase. The direction of the molecular tilt varies from layer to layer. The molecules are drawn in different shades of gray in order to distinguish them. 


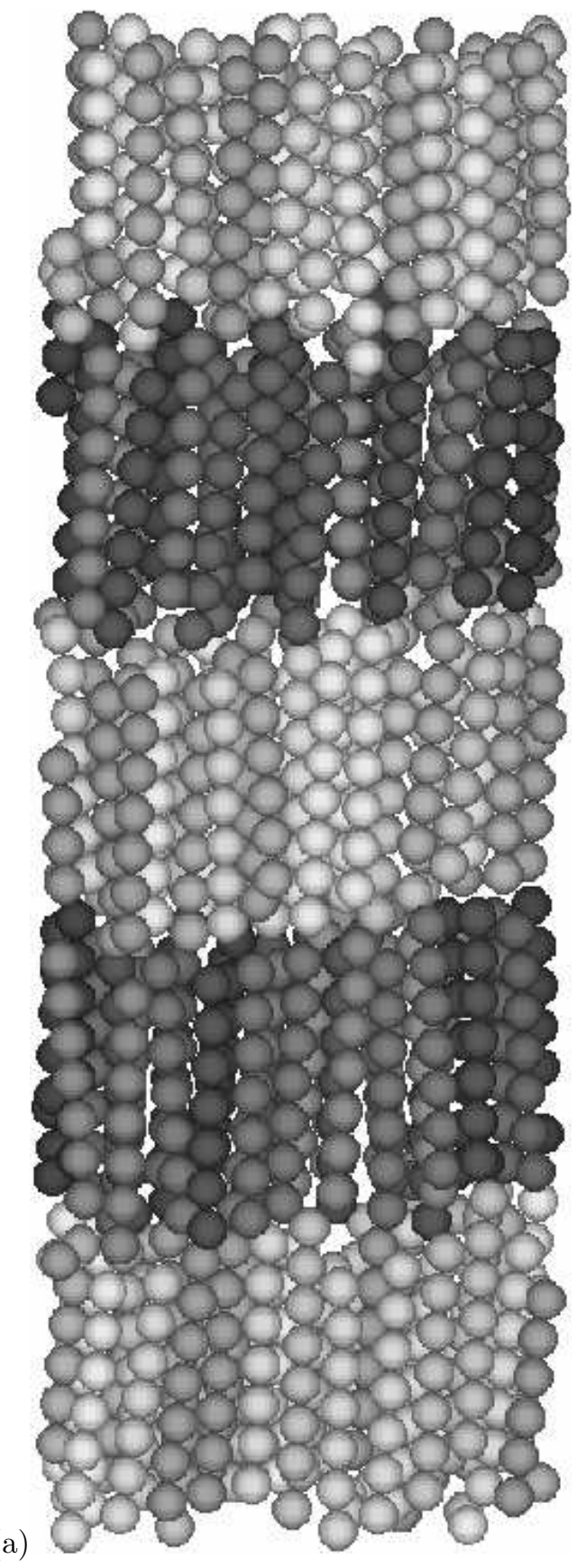

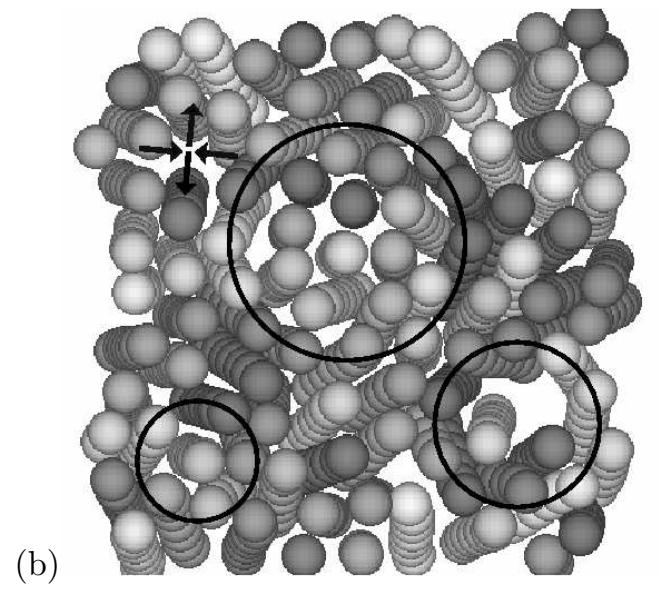

FIG. 3. For the molecules with $\theta=5^{\circ}$, the simulations show a SmA phase at $k_{B} T=1.3$. (a) Side view. (b) Top view of a single smectic layer. The circles indicate three vortices with positive topological charge (two right-handed and one left-handed), and the arrows indicate one vortex with negative topological charge. 


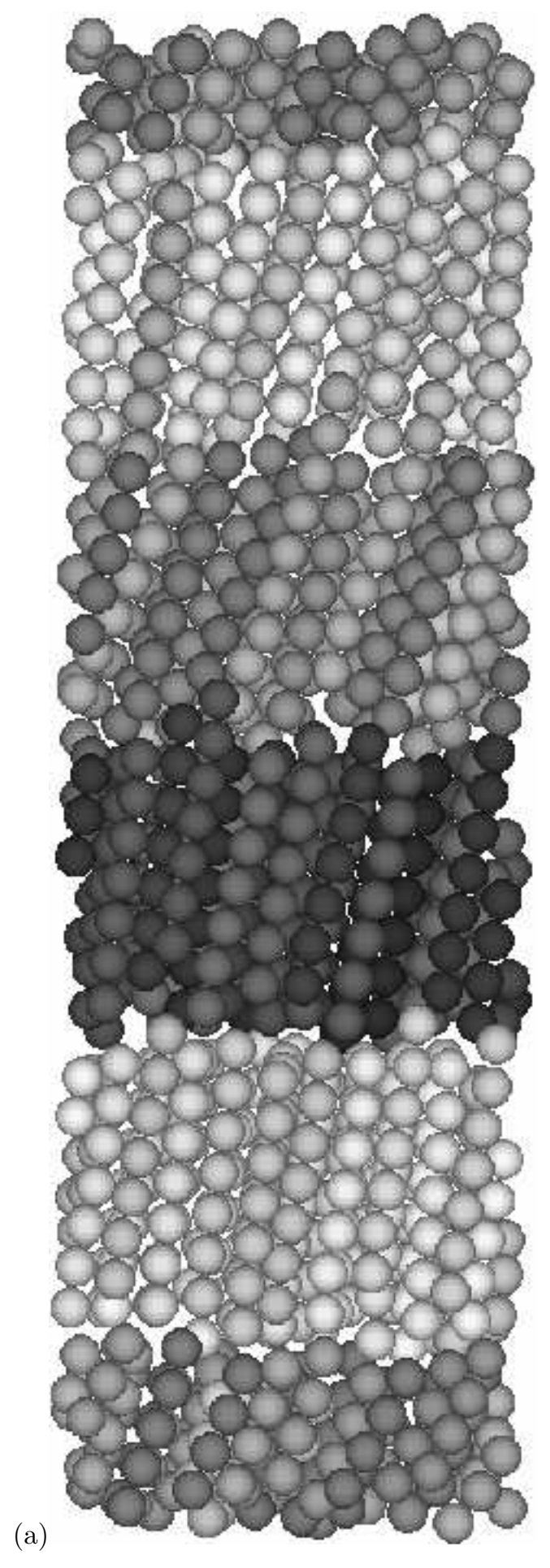

(b)

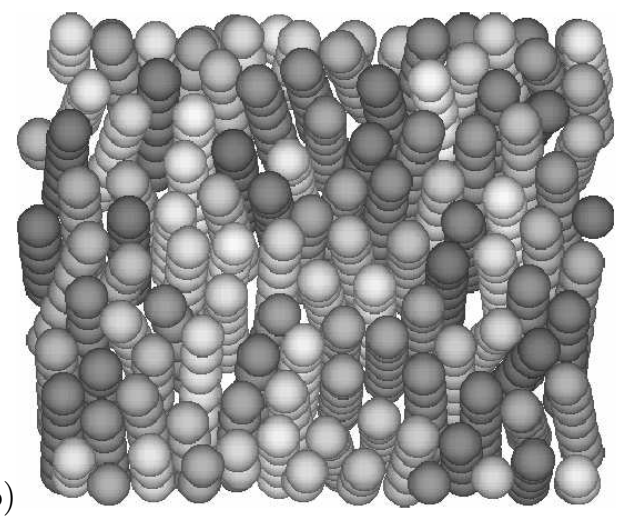

FIG. 4. Under an applied electric field in the $x$ direction, the molecules tilt with respect to the smectic layer normal, showing an electroclinic effect. This picture shows the SmA system at $k_{B} T=1.3$ under a strong field $E=10$, with an induced tilt of approximately $19^{\circ}$. (a) Side view. (b) Top view of a single smectic layer, showing that the vortices have disappeared.

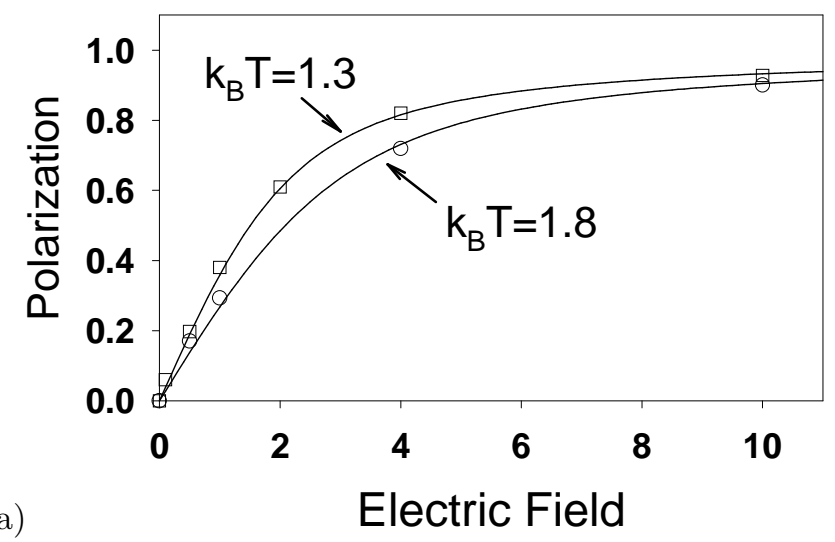

(b)

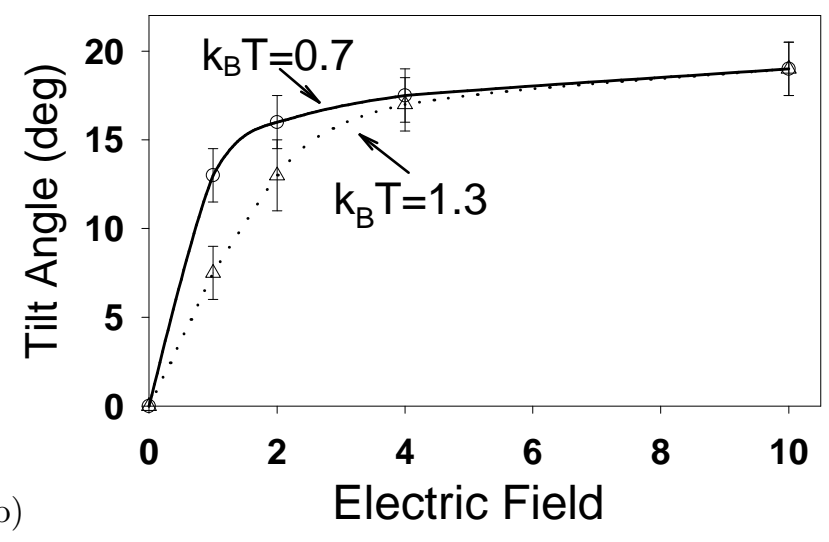

FIG. 5. (a) Induced polarization of the SmA phase as a function of applied electric field, compared with the prediction of Eq. (6) from a two-dimensional spin model. Polarization is measured in units of the molecular dipole moment $p$, temperature in units of the Lennard-Jones parameter $\epsilon$, and electric field in units of $\epsilon / p$. (b) Induced tilt angle of the SmA phase as a function of applied electric field. In part b, the lines are guides to the eye. 


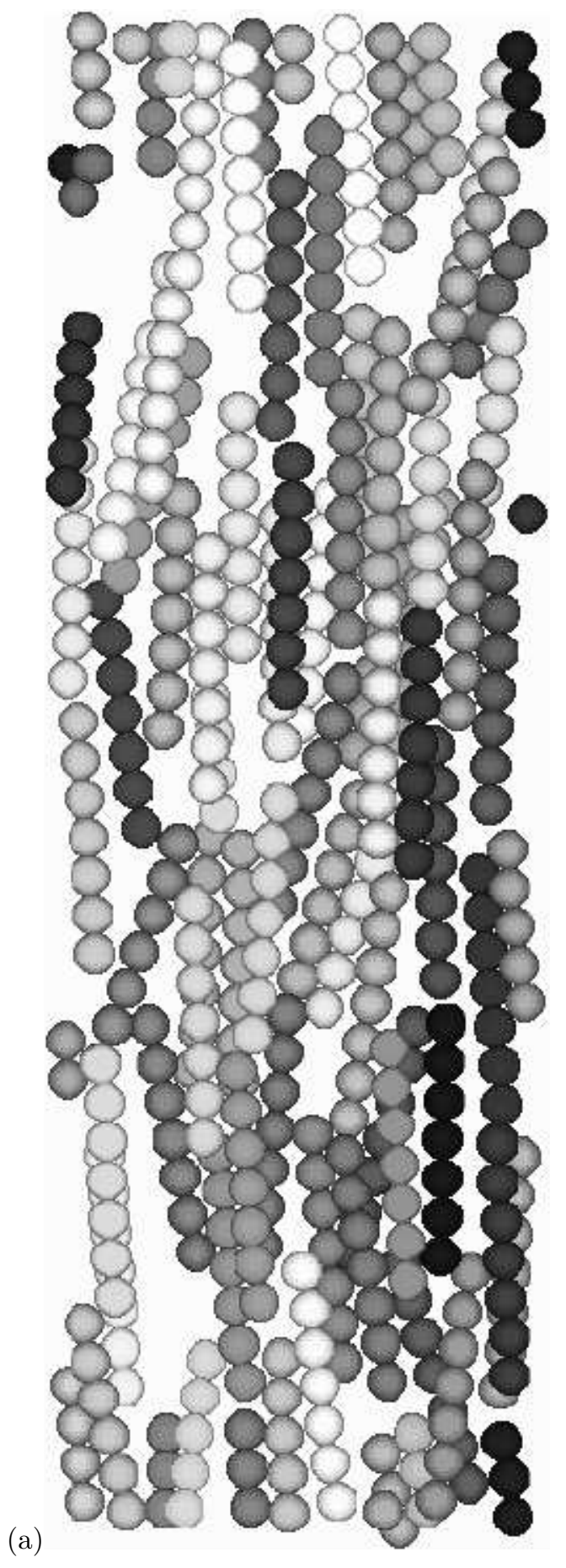

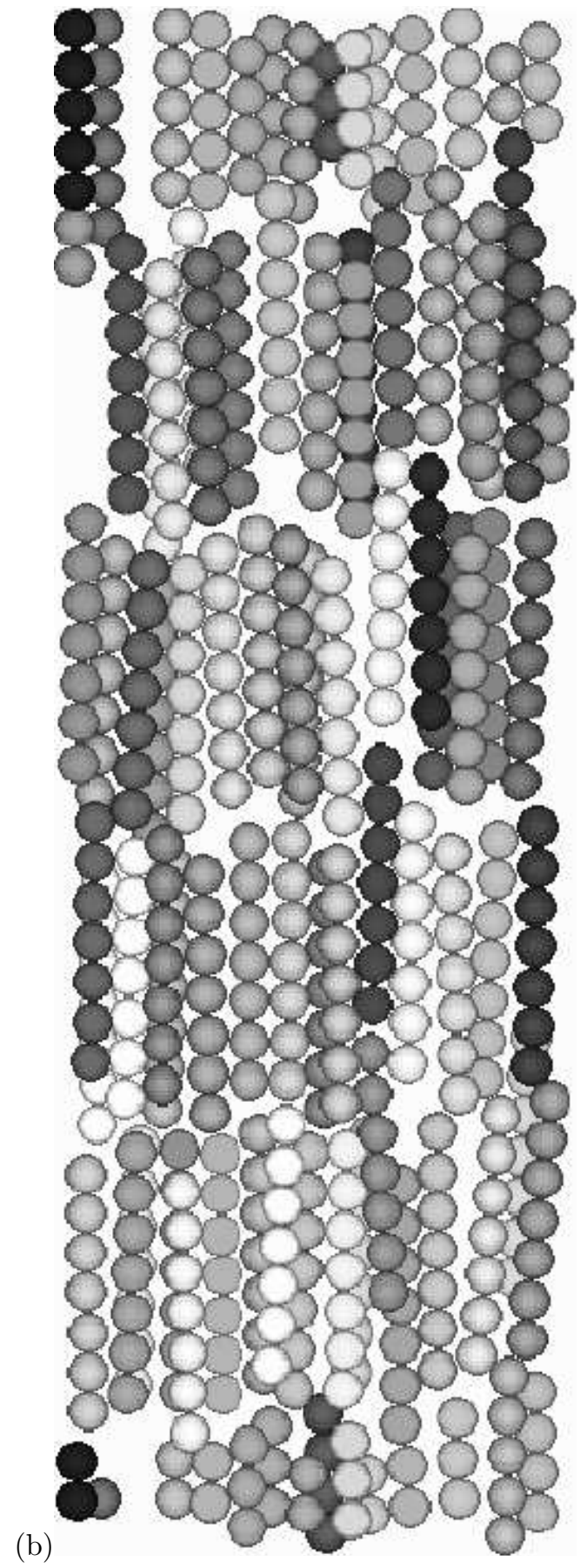

FIG. 6. (a) At $k_{B} T>3.0$, the SmA phase melts into a nematic phase with no positional correlations but with very high orientational correlations. This image shows a side view of a slice through the nematic phase at $k_{B} T=3.3$. (b) Under a strong electric field, the nematic phase regains smectic order. This image shows a side view of a slice through the system at $E=10$ and $k_{B} T=3.3$. Five smectic layers can be seen. 